\title{
AN ERROR ESTIMATE FOR CONTINUED FRACTIONS
}

\author{
JOHN GILL
}

\begin{abstract}
New and improved truncation error bounds are derived for continued fractions $K\left(a_{n} / 1\right)$, where $a_{n} \rightarrow 0$. The geometrical approach is somewhat unusual in that it involves both isometric circles and fixed points of bilinear transformations.
\end{abstract}

Continued fractions of the form

$$
\frac{a_{1}}{1}+\frac{a_{2}}{1}+\ldots
$$

where $a_{n} \in \mathbf{C}, a_{n} \neq 0$ for $n \geqslant 1$, and $a_{n} \rightarrow a$, are called limit periodic. In this paper we shall obtain new and improved a priori error bounds for both the classical continued fraction (1) and its modified form [2] in the case $a=0$.

Recently obtained results in error analysis/acceleration by Thron and Waadeland $[6,7]$ apply only in the case $a \neq 0$, and the author's results along these lines [2] require the sort of bounds described in the present paper.

(1) may be perceived as a composition of bilinear transformations in the following way: Set $t_{n}(z)=a_{n} /(1+z)$ and $T_{n}(z)=t_{1} \circ \cdots \circ t_{n}(z)$ for $n \geqslant 1$. The $n$th approximant of (1) is then $T_{n}(0)$.

The isometric circle of $t_{n}(z)$ is defined as $I_{n}=\left\{z:|z+1|=\sqrt{\left|a_{n}\right|}\right\}, n \geqslant 1$. By an application of $t_{n}$, distances outside $I_{n}$ are diminished, and those inside $I_{n}$ are increased [1]. $t_{n}$ operating on a circle exterior to $I_{n}$ contracts the circle through an inversion in $I_{n}$ and reflects and rotates the resulting circle. If $t_{n}$ has an attractive fixed point $\alpha_{n}$, and $\alpha_{n}$ lies inside the original circle, it also lies inside the transformed circle.

Let us assume that $\left|\arg \left(a_{n}+1 / 4\right)\right|<\pi$ and $\operatorname{Re}\left(\sqrt{a_{n}+1 / 4}\right)>0$. Then $\alpha_{n}=$ $-1 / 2+\sqrt{a_{n}+1 / 4}$ is the attractive fixed point of $t_{n}$ (i.e., $t_{n}$ has two distinct fixed points $\alpha_{n}$ and $\beta_{n}$ and $\left.\left|\alpha_{n}\right|<\left|\beta_{n}\right|\right)$. We write

$$
\begin{gathered}
A_{n}=\sup _{m \geqslant n} \sqrt{\left|a_{m}\right|}, \quad A=A_{1}, \\
P_{n}=\frac{2-A}{2-A-2 A_{n}^{2} / A}, \quad \text { and } \varepsilon_{n}=P_{n} \cdot \sup _{m \geqslant n}\left|-\frac{1}{2}+\sqrt{a_{m}+\frac{1}{4}}\right|
\end{gathered}
$$

for $n \geqslant 1$.

Received by the editors May 20, 1984.

1980 Mathematics Subject Classification. Primary 40A15.

(C) 1986 American Mathematical Society $0002-9939 / 86 \$ 1.00+\$ .25$ per page 
With reference to (1) we have

THEOREM. If

(i) $a_{n} \rightarrow 0$,

(ii) $A<2 / 3$, and

(iii) $\sup _{m \geqslant n}\left|-\frac{1}{2}+\sqrt{a_{m}+\frac{1}{4}}\right|<(1-A) / P_{1}, n \geqslant 1$ are satisfied,

then (1) converges to a value $T$, and

$$
\begin{aligned}
\left|T_{n}\left(\mu_{n}\right)-T\right| & <2 \varepsilon_{n} \prod_{m=1}^{n}\left(\frac{A_{m}}{1-\varepsilon_{m}}\right)^{2}, . \quad n \geqslant 1, \text { where } \mu_{n}=0 \text { or } \mu_{n}=\alpha_{n+1} \\
& =\left|-\frac{1}{2}+\sqrt{a_{n+1}+\frac{1}{4}}\right| .
\end{aligned}
$$

Proof. The condition $A<2 / 3$ implies $\sqrt{\left|a_{n}\right|}<1$ for $n \geqslant 1$. Thus $a=\alpha=0$ lies outside $I_{n}$ for $n \geqslant 1$. We seek disks $C_{n}=\left\{z:|z| \leqslant R_{n}\right\}$ such that $C_{n+1} \subset C_{n}$, $t_{m}\left(C_{n}\right) \subseteq C_{n}$ for $m \geqslant n, C_{n}$ lies outside $I_{n}, \alpha_{m}$ lies inside $C_{n}$ for $m \geqslant n$, and $\operatorname{Rad}\left[T_{n}\left(C_{n}\right)\right] \rightarrow 0$. With the notation

$$
M_{n}=\sup _{m \geqslant n}\left|\alpha_{m}\right| \text { and } Y_{n}=\sup _{m \geqslant n}\left\{s_{m}: s_{m}=\operatorname{Rad}\left[t_{m}\left(C_{n}\right)\right]\right\},
$$

the following two conditions reflect these criteria:

I. $R_{n} \geqslant M_{n}+2 Y_{n}$, and

II. $R_{n}<1-A \leqslant 1-A_{n}$ for $n \geqslant 1$ (see the Figure).

The transformation $t_{m}\left(C_{n}\right)$ involves an inversion in $I_{m}$, so that

$$
s_{m}=\left(\sqrt{\left|a_{m}\right|}\right)^{2} /\left[\left(1-R_{n}\right)\left(1+R_{n}\right)\right], \quad m \geqslant n .
$$

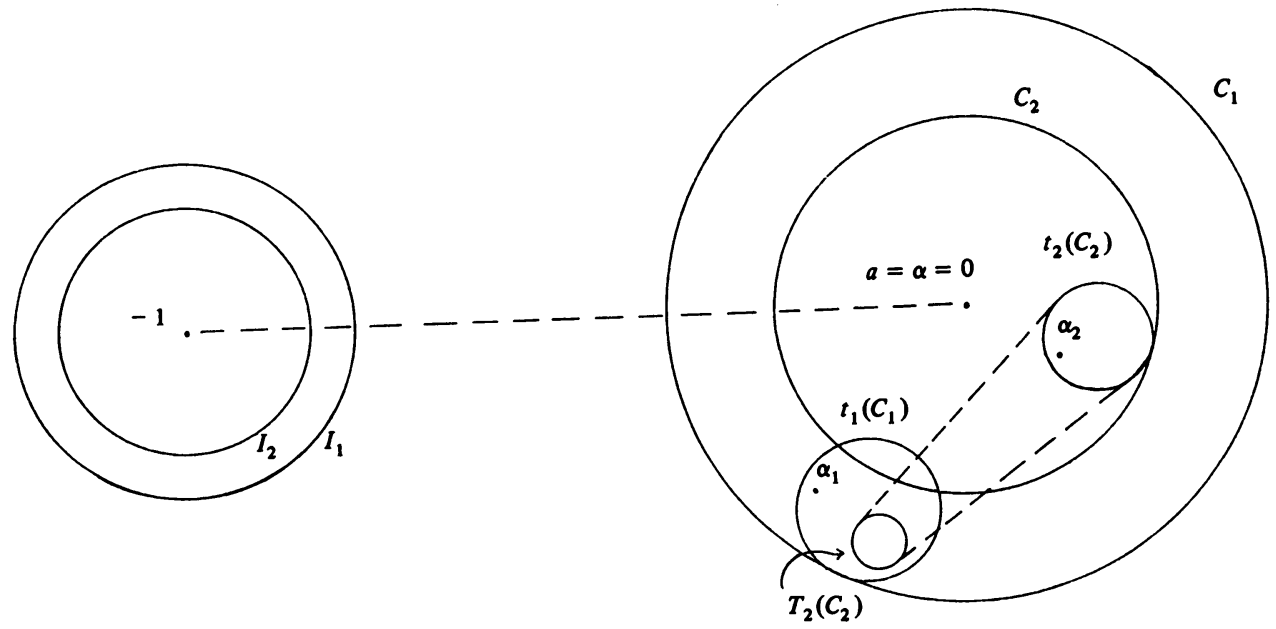


This implies $Y_{n}=\left[A_{n}^{2} /\left(1-R_{n}\right)^{2}\right] \cdot R_{n}$. Assuming for the moment that $R_{n}<1-A$, we find that $Y_{n}<\left[A_{n}^{2} /\left(2 A-A^{2}\right)\right] \cdot R_{n}$. Therefore, we define $R_{n}$ in the following way:

$$
R_{n}:=M_{n}+2 A_{n}^{2} R_{n} /\left(2 A-A^{2}\right)>M_{n}+2 Y_{n},
$$

thus insuring I. This gives $R_{n}=M_{n} \cdot P_{n}$, where $P_{n} \leqslant(2-A) /(2-3 A)=P_{1}$.

Condition II is guaranteed by requiring

$$
M_{n}<(1-A) / P_{1} \leqslant(1-A) / P_{n}, \quad n \geqslant 1 .
$$

It follows that $R_{n}(\downarrow)$ to 0 , since $M_{n}(\downarrow)$ to 0 and $P_{n}>0$ decreases monotonically. If $C$ is a circle of radius $Y$ contained in $C_{n}$, then the radius $Y^{*}$ of the transformed circle $t_{n}\left(C_{n}\right)$ satisfies

$$
Y^{*}<\left[\frac{\sqrt{\left|a_{n}\right|}}{1-R_{n}}\right]^{2} \cdot Y \leqslant \frac{A_{n}^{2}}{\left(1-R_{n}\right)^{2}} \cdot Y .
$$

Therefore,

$$
\begin{gathered}
\operatorname{Rad}\left[t_{n}\left(C_{n}\right)\right]<\left(\frac{A_{n}}{1-R_{n}}\right)^{2} \cdot R_{n}, \\
\operatorname{Rad}\left[t_{n-1} \circ t_{n}\left(C_{n}\right)\right]<R_{n} \prod_{m=n-1}^{n}\left(\frac{A_{m}}{1-R_{m}}\right)^{2}, \\
\vdots \\
\operatorname{Rad}\left[T_{n}\left(C_{n}\right)\right]<R_{n} \prod_{m=1}^{n}\left(\frac{A_{m}}{1-R_{m}}\right)^{2},
\end{gathered}
$$

where $\lim _{m \rightarrow \infty}\left[A_{m} /\left(1-R_{m}\right)\right]=0$.

Now, since $T_{n}(0), T_{n}\left(\alpha_{n+1}\right) \in T_{n}\left(C_{n}\right)$ and $\lim T_{n}\left(\mu_{n}\right)=T$ if $\mu_{n} \rightarrow 0$ [4], we obtain the conclusion of the theorem.

EXAMPLE.

$$
\frac{11 / 10^{2}}{1}+\frac{21 / 20^{2}}{1}+\cdots+\frac{(10 n+1) /(10 n)^{2}}{1}+\ldots
$$

Bounds for $\left|T_{n}(0)-T\right|$ are

\begin{tabular}{ccccc}
$n$ & Actual Error & Theorem est. & Theorem 1 [3] est. & Theorem 2.1 [5] est. \\
\hline 1 & $5.3 \times 10^{-3}$ & $5.3 \times 10^{-2}$ & $\mathrm{~N} / \mathrm{A}$ & $\mathrm{N} / \mathrm{A}$ \\
3 & $4.1 \times 10^{-6}$ & $4.2 \times 10^{-5}$ & $5.4 \times 10^{-3}$ & $3.5 \times 10^{-3}$ \\
5 & $1.2 \times 10^{-9}$ & $1.5 \times 10^{-8}$ & $7.1 \times 10^{-6}$ & $2.0 \times 10^{-4}$
\end{tabular}




\section{BIBLIOGRAPHY}

1. L. R. Ford, Automorphic functions, McGraw-Hill, New York, 1929, pp. 23-30.

2. J. Gill, Converging factors for continued fractions $K\left(a_{n} / 1\right), a_{n} \rightarrow 0$, Proc. Amer. Math. Soc. 84 (1982), 85-88.

3. Truncation error analysis for continued fractions $K\left(a_{n} / 1\right)$, where $\sqrt{\left|a_{n}\right|}+\sqrt{\left|a_{n-1}\right|}<1$, Lecture Notes in Math., vol. 932, Springer-Verlag, Berlin and New York, 1982, pp. 71-73.

4. __ Modifying factors for sequence of linear fractional transformations, Norske Vid. Selsk. Skr. (Trondheim) 3 (1978), 1-7.

5. W. Jones and R. Snell, Truncation error bounds for continued fractions, SIAM J. Numer. Anal. 6 (1969), 210-221.

6. W. Thron and W. Waadeland, Accelerating convergence of limit periodic continued fractions $K\left(a_{n} / 1\right)$, Numer. Math. 34 (1980), 155-170.

7. Truncation error bounds for limit periodic continued fractions, Math. Comp. 40 (1983), 589-597.

Department of Mathematics, University of Southern Colorado, Pueblo, Colorado 81001 